\title{
Research Article \\ Fuzzy Timing Petri Net for Fault Diagnosis in Power System
}

\author{
Alireza Tavakholi Ghainani, ${ }^{1}$ Abdullah Asuhaimi Mohd Zin, ${ }^{2}$ \\ and Nur 'Ain Maiza Ismail' \\ ${ }^{1}$ Faculty of Electrical Engineering, Islamic Azad University, Najafabad Branch, \\ No 252 Khaghani Street, 8175848591 Isfahan, Iran \\ ${ }^{2}$ Faculty of Electrical Engineering, Universiti Teknologi Malaysia, 81300 Johor Bahru, Johor, Malaysia \\ Correspondence should be addressed to Nur 'Ain Maiza Ismail, maiza@fke.utm.my
}

Received 8 March 2012; Accepted 27 June 2012

Academic Editor: Zheng-Guang Wu

Copyright ( 2012 Alireza Tavakholi Ghainani et al. This is an open access article distributed under the Creative Commons Attribution License, which permits unrestricted use, distribution, and reproduction in any medium, provided the original work is properly cited.

\begin{abstract}
A model-based system for fault diagnosis in power system is presented in this paper. It is based on fuzzy timing Petri net (FTPN). The ordinary Petri net (PN) tool is used to model the protective components, relays, and circuit breakers. In addition, fuzzy timing is associated with places (token) / transition to handle the uncertain information of relays and circuits breakers. The received delay time information of relays and breakers is mapped to fuzzy timestamps, $\pi(\tau)$, as initial marking of the backward FTPN. The diagnosis process starts by marking the backward subFTPNs. The final marking is found by going through the firing sequence, $\sigma$, of each sub-FTPN and updating fuzzy timestamp in each state of $\sigma$. The final marking indicates the estimated fault section. This information is then in turn used in forward FTPN to evaluate the fault hypothesis. The FTPN will increase the speed of the inference engine because of the ability of Petri net to describe parallel processing, and the use of time-tag data will cause the inference procedure to be more accurate.
\end{abstract}

\section{Introduction}

A rapid and correct fault diagnosis is crucial for power system restoration. However, as the complexity of power system increases, fault diagnosis, especially in complicated faults or incorrect operation of protective devices, becomes a very difficult task in the limited short time. This situation has made it necessary to develop intelligent systems to support operators in their decision making process. Over the last two decades different artificial intelligent (AI) approaches have been proposed for fault diagnosis in power system. Most attempts to date have relied on the use of expert system [1] or neural network [2] technology. Expert-systembased approaches have been the most successful so far, while neural-network-based methods 
continue to improve their performance. Previous reported expert systems for fault diagnosis use either rule-based or model-based approach [3]. The first approach may work well only in simple fault cases. However, to diagnose a fault in complicated cases, it needs a huge number of rules to describe the complicated protection system behavior. As a result, the acquisition and maintenance of such a system is tedious and difficult [3].

On the other hand, model-based diagnostic (MBD) methods are suitable to network fault diagnosis because the power systems and protective relays can be modeled as discrete event systems. MBD covers a wide range of fault scenarios than heuristic reasoning because it is based on the system behavioral analysis. It can detect malfunctioning equipment in the early stages [4]. Nevertheless, the model-based system requires more inference time. As a result, there is a need to enhance speed and performance of diagnoses system. Parallel inference processing and time sequence information of protective relays and circuit breakers is important factor for reducing fault diagnosis processing time [3]. This is so because parallel processing increases the inference procedure and real-time availability of the relay information allows expert systems to reduce the number of hypotheses [4].

One of the powerful tools for modeling parallel processing is Petri net [5]. There have been some proposed model-based systems using Petri net and colored and timed Petri net for faster inference [6, 7]. In [6] final marking of forward and backward Petri nets model is compared to make a decision for faulted section area. However, timestamp of protective devices has not been considered on that model and the model which is proposed in [7] cannot handle the uncertain and missing data. There have also been works on expert systems that use time-tag information of actuated relays and tripped circuit breakers through sequence event recorder (SER) in fault diagnosis [8].

This paper proposes fuzzy timing Petri net to handle uncertain information of protective device and to overcome the drawbacks of previous works. Petri nets have also been successfully applied in power system for verification of concurrent switching sequences [9] and modeling of transmission line protection relaying scheme [10].

The paper is organized as follows. In the next section Petri net will be introduced. A brief and concise description of the fuzzy timing petri net (FTPN) will be given in Section 3. Diagnosis process is described in Section 4. In Section 5, the proposed FTPN is used for diagnosing fault in a simple and typical line. The application will be presented in Section 7. The final section is conclusion.

\section{Petri Nets}

Petri nets (PNs), as a graphical and mathematical tool, provide a uniform environment for modeling and design of discrete event systems. It is a particular kind of bipartite directed graphs populated by three objects. These objects are places, transitions, and directed arcs connecting places to transitions and transitions to places. Pictorially, places are depicted by circles and transitions by bars [5].

The ordinary Petri nets do not include any concept of time explicitly. With this class of nets, it is only possible to describe the logical structure of the modeled system, but not its time evolution. Responding to the need for the temporal performance of discrete-event systems and modeling concurrent systems with time constraints, various timed extensions of Petri nets have been proposed by attaching timing constraints to transitions, places, and/or $\operatorname{arcs}[5]$.

Later, other researchers introduced fuzzy Petri net for knowledge representation to deal with fuzzy production rules [11] and fuzzy timing Petri net (FTPN) for performance, 
evaluation, and specification of dynamic concurrent system $[12,13]$ under uncertainty and imprecision.

\section{Fuzzy Timing Petri Net and Extended Fuzzy Timing Petri Net}

Fuzzy-timing Petri net (FTPN) has been proposed by Zhou and Murata [12] and is defined as follows.

The static structure of FTPN is a five-tuple structure, $N=(P, T, A, D, \mathrm{FT})$ where $P=$ $\left\{p_{1}, p_{2}, \ldots, p_{n}\right\}$ is a finite set of places, $T=\left\{t_{1}, t_{2}, \ldots, t_{m}\right\}$ is a finite set of transitions, $A \subseteq$ $(P \times T) \cup(T \times P)$ is a set of arcs (flow relation), $D$ is a set of all fuzzy delays $d_{t p}(\tau)$ associated with arcs $\subseteq(T \times P)$, and FT is a set of all fuzzy timestamp, where a fuzzy timestamps, $\pi(\tau) \in$ FT is associated with each token and each place. It is a fuzzy time function or possibility distribution giving the numerical estimate of the possibility that a particular token arrives at time $\tau$ in a particular place.

The extended fuzzy-timing Petri net (EFTPN) model is a FTPN with the default value of $d_{t p}(\tau)$ being $(0,0,0,0)$ and with additional function CT :T $\rightarrow Q^{+} \times Q^{+} \times\left(Q^{+} \cup \infty\right)$, which is a mapping from transition $T$ to firing intervals with possibility $p$, that is, each transition is associated with a firing interval, $p[a, b],(a \leq b)$, where the default interval is $1[0,0]$ (a transition definitely fires as soon as it is enabled) (possibility $p \in[0,1]$ ). $P$ is 1 if transition $t$ is not in conflict with any other transition. When different chances are to be assigned to transitions in structural conflict, $P$ can be less than $1 . Q^{+}$is set of positive rational numbers.

The dynamic evolution of marking in an FTPN is the same as that of an ordinary PN except that fuzzy timestamps $\pi(\tau)$, fuzzy enabling times $e(\tau)$, and fuzzy occurrence time $o(\tau)$ need to be computed and updated each time when a transition firing (atomic action) occurs. Fuzzy enabling time $e_{t}(\tau)$ of transition $t$ is the possibility distribution of latest arrival time among the arrival times of all tokens in input places of $t$ that are necessary to enable the transition $t$ in the untimed case and is given by

$$
e_{t}(\tau)=\text { latest }\left\{\pi_{i}(\tau), i=1,2, \ldots, n\right\}
$$

Fuzzy occurrence time $O_{t}(\tau)$ of transition $t$ is the possibility distribution of the time at which the transition $t$ starts firing and is given by

$$
O_{t}(\tau)=\min \left\{e_{t}(\tau), \text { earliest }\left\{e_{i}(\tau), i=1,2, \ldots m\right\}\right\}
$$

The fuzzy timestamp $\pi_{t p}(\tau)$, the possibility distribution of the time at which a token arrives in an output place of $t$, is given by the extended addition of $O_{t}(\tau)$ and $d_{t p}(\tau)$ or

$$
\pi_{t p}(\tau)=O_{t}(\tau) \oplus d_{t p}(\tau)
$$

Here $\pi_{t p}(\tau)$ is updated fuzzy timestamps in an FTPN. When there are $m$ transitions in conflict enabled with their fuzzy enabling times, $e_{i}(\tau), i=1,2, \ldots . m$, and $\mathrm{CT}\left(t_{i}\right)=p_{i}\left[a_{i}, b_{i}\right]$, then fuzzy occurrence time $O_{t}(\tau)$ of transition $\mathrm{t}$ is computed as follows:

$$
O_{t}(\tau)=\min \left\{e_{t}(\tau) \oplus p_{t}\left(a_{t}, a_{t}, b_{t}, b_{t}\right), \text { earliest }\left\{e_{i}(\tau) \oplus p_{i}\left(a_{i}, a_{i}, b_{i}, b_{i}\right), i=1,2, m\right\}\right\} .
$$




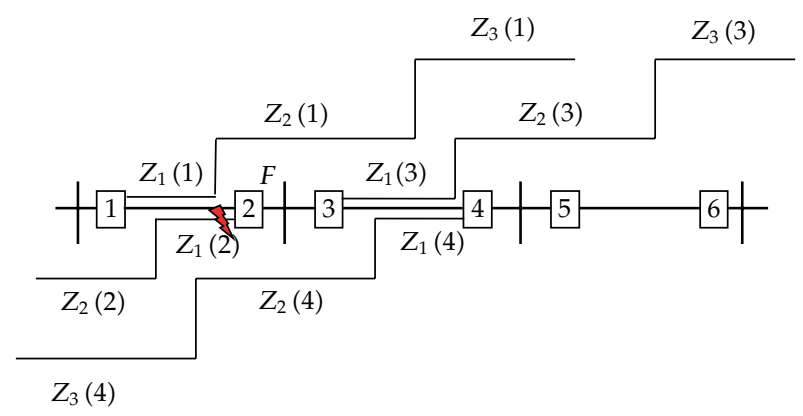

Figure 1: A simple and typical transmission line.

\section{Diagnosis Process}

In the following discussion it is assumed that the protective devices have arrived in their final status. The general philosophy of diagnosis task is based on model-based reasoning: the comparison between the observed and predicted behaviors of the system [14-16]. Diagnosis is performed in two-step reasoning process. The first step is based on forward reasoning (data driven). Having the final status of protective devices, the initial marking of the backward FTPN is performed by assigning fuzzy time function $\pi(\tau)$ to relevant places. That is to say, timestamps information of relays and breakers is used as the initial fuzzy timestamps $\pi_{0}(\tau)$. In other words, $\pi_{0}(\tau)$ is the numerical estimate of possibility that a particular protective device has been operated. Processing the FTPN as a forward reasoning to get final marking would get the fault hypotheses. Indeed in the first step of diagnosis, both the candidates of faulted section and estimated time that fault has been cleared by protective devices are derived.

Fault simulation process takes place in the second step of diagnosis task and is based on backward reasoning (goal driven). The predicate behavior of protective devices, in the case of occurring fault, is modeled by the forward FTPN. The fuzzy timestamp of token arriving at the final place of backward FTPN is compared with fuzzy timestamp of token in the final state of forward FTPN.

A default threshold value, $\lambda$, is used to validate the discrepancy of two fuzzy timestamps. If discrepancy of two fuzzy timestamps is larger than threshold value, then the fault candidate is assumed to be correct. Otherwise the simulation process is repeated again by executing the forward FTPN by assuming the malfunction of appropriate relay. For instance, by exchanging the possibility of transition $t_{2}$ and $t_{3}$ in Figure 2 the malfunction of relay $R_{1}$ is simulated.

\section{Example}

For illustration purposes, consider a simple and typical transmission line depicted in Figure 1. Suppose a fault has occurred on point $F$. Furthermore, assume that signals have been received and recorded with precise time tags or in a chronological order and available through SER.

The forward and backward FTPN models with main protection $\left(\mathrm{CB}_{2}\right)$ and primary backup protection $\left(\mathrm{CB}_{1}, \mathrm{CB}_{4}\right)$ for this point are shown in Figure 2 and Figure 4, respectively.

In Figure 2, the token in place $P_{1}$ shows absence of the fault, $F$, and $P_{5}, P_{9}$, and $P_{13}$ represent readiness of the relays $R_{2}, R_{1}$, and $R_{4}$, respectively, Places $P_{16}, P_{17}$, and $P_{18}$ 


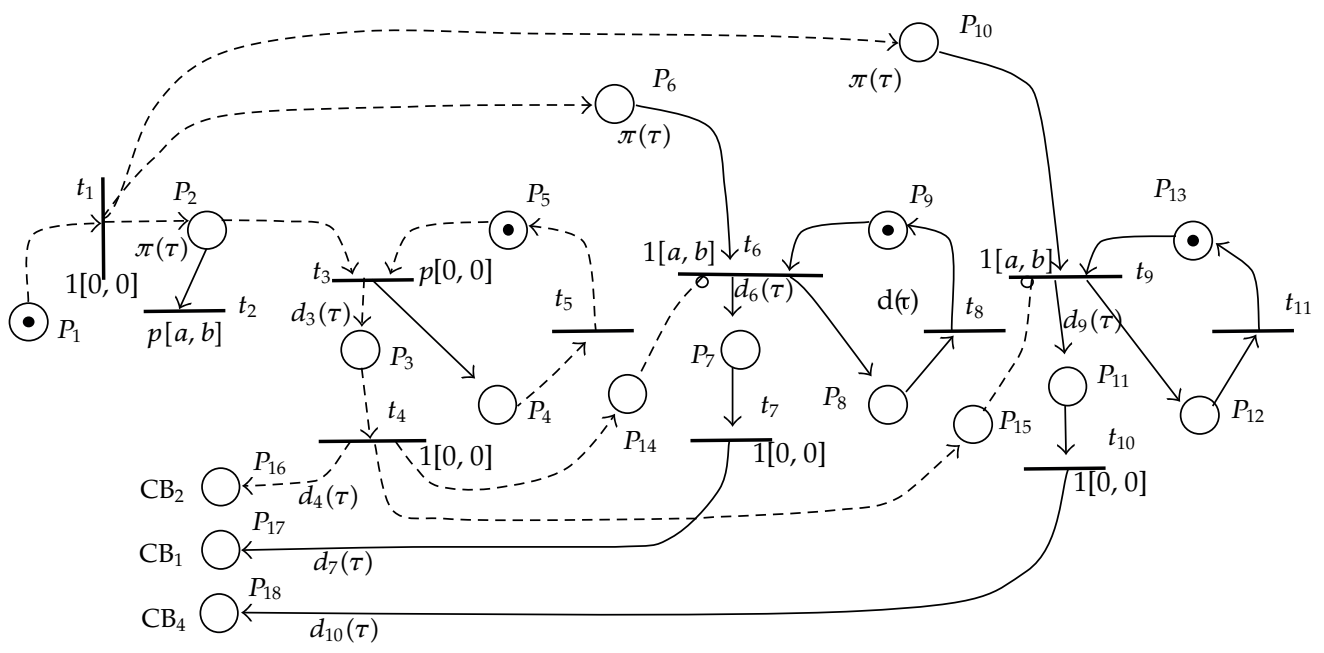

Figure 2: Forward FTPN model for fault at $F$ point in Figure 1. $\sigma_{1}: M_{0}\left[t_{1}\right\rangle M_{1}\left[t_{3}\right\rangle M_{2}\left[t_{4}\right\rangle M_{3}\left[t_{5}\right\rangle M_{4}, \sigma_{2}$ :

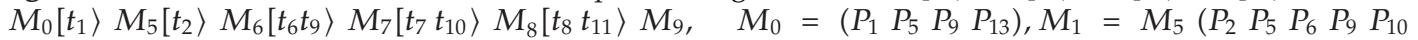
$\left.P_{13}\right), \quad M_{2}=\left(\begin{array}{llllll}P_{3} & P_{4} P_{6} P_{9} P_{10} P_{13}\end{array}\right), \quad M_{3}=\left(\begin{array}{lllllll}P_{4} P_{6} P_{9} P_{10} P_{13} P_{14} P_{15} P_{16}\end{array}\right), M_{4}=\left(\begin{array}{lllll}P_{5} & P_{6} P_{9} P_{10} P_{13}\end{array}\right.$ $\left.P_{14} P_{15} P_{16}\right), \quad M_{6}=\left(\begin{array}{llll}P_{5} & P_{6} P_{9} P_{10} P_{13}\end{array}\right), M_{7}=\left(P_{5} P_{7} P_{8} P_{11} P_{12}\right), M_{8}=\left(\begin{array}{llll}P_{5} P_{8} P_{12} P_{17} P_{18}\end{array}\right), M_{9}=$

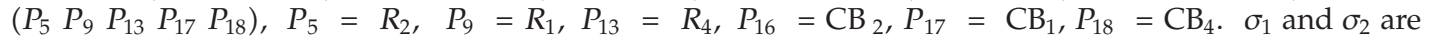
the firing sequences, in the case of correct actuated and nonactuated of relay $R_{2}$, respectively, $M_{0}$ to $M_{9}$ are marking states of the FPTN.

correspond to circuit breakers $\mathrm{CB}_{2}, \mathrm{CB}_{1}$, and $\mathrm{CB}_{4}$, respectively, The occurrence of $F$ is represented by the transition $t_{1}$, which deposits a token in places $P_{2}, P_{6}$, and $P_{10}$ to indicate that the fault is present.

In this case, transitions $t_{3}, t_{6}$, and $t_{9}$ are enabled and can fire within their interval time. This corresponds to sensing the fault by relay $R_{2}, R_{1}$, and $R_{4}$. However, transitions $t_{7}$ and $t_{10}$ will be fired after transition $t_{3}$ because their firing interval is later than $t_{3}$. The static default of firing interval of transition $t_{3}$ is $[0,0]$. Firing transitions $t_{3}, t_{6}$, and $t_{9}$ correspond to sending trip signals and transitions $t_{4}, t_{7}$, and $t_{10}$ correspond to opening the circuit breakers $\mathrm{CB}_{2}, \mathrm{CB}_{1}$, and $\mathrm{CB}_{4}$, respectively.

A fuzzy delay $d_{t p}(\tau)$ is associated with $\operatorname{arcs}(t, p)$ from transitions $t_{4}, t_{7}$, and $t_{10}$ to places $P_{16}, P_{17}$, and $P_{18}$, respectively, to map the operating time of $\mathrm{CB} s$. The $d_{t p}(\tau)$ of other arcs are set to $(0,0,0,0)$, which means that transitions connected to these arcs fire and the token will be available to their corresponding output place immediately. The sink transitions $t_{2}$ is fired in the case of malfunction of relay $R_{2}$. Since backup relays send trip signal after main relays, the firing transitions of the FTPN corresponding to these relays should be in correct sequence.

To do this, a static time interval $1[a, b](a \leq b)$ is assigned to the transitions $t_{6}$ and $t_{9}$ to ensure that these transitions will be fired after transitions $t_{3}$ and $t_{4}$. Moreover, in the case of malfunction of $\mathrm{CB}_{2}$, places $P_{14}$ and $P_{15}$ will not get tokens. Therefore, transitions $t_{6}$ and $t_{9}$ can fire within their firing intervals. The firing sequences and its marking of the forward FTPN are shown in the bottom of Figure 2.

The backward FTPN consists of three sub-FTPN modules (see Figure 4). Each of the sub-FTPNs corresponds to one CB and its corresponding relay protection module. There are three kinds of places in this FTPN: those which get marking in the case of receiving signals (shown with a circle), the second type that get token in the case of nonreceiving signals from 


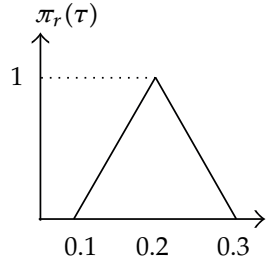

(a)

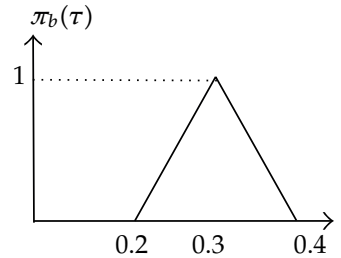

(b)

Figure 3: Two typical fuzzy time functions (a) for delay time of relay $R_{2}$ and (b) for delay time of breaker $\mathrm{CB}_{2}$.

CBs and relays (shown with two circles), and the third one which are used as auxiliary places (shown also with a circle).

In Figure 2, places $P_{1}, P_{4}, P_{9}, P_{12}, P_{17}$, and $P_{20}$ correspond to $\mathrm{CB}_{1}, R_{1}, \mathrm{CB}_{2}, R_{2}, \mathrm{CB}_{4}$, and $R_{4}$, respectively. In the case of non-receiving signal from relay or $\mathrm{CB}$, the places indicated by two circles get tokens.

As previously mentioned, suppose a fault has occurred at point $F$ and information received from relays and breakers with their time delay is $R_{2}(0.2 s)$ and $\mathrm{CB}_{2}(0.3 s)$. Diagnosing process starts by marking appropriate places of the backward sub-FTPN (Figure 4) and assigning each token with fuzzy time function. The goal is to find the fuzzy time function of final state of the backward FTPN in its firing sequences.

To do this, first fuzzy enabling time of transition $t_{11}$ is calculated by (3.1). Then the fuzzy occurrence time of $t_{11}$ is found by (3.2). The next step is to compute fuzzy timestamp of place $P_{11}$. It is calculated by (3.3). The same procedure is done for the next transitions / places in the firing sequences $\sigma_{1}$ (shown at the bottom of Figure $4(\mathrm{a})$ ) to reach the place $F$.

At this stage of diagnosis, the fuzzy timestamps at the place $F$ are compared with the simulation, result of Figure 2. If discrepancy of two fuzzy timestamps is larger than threshold value and receiving data is compatible with simulation, then the fault candidate is assumed to be correct. Otherwise the simulation process is repeated. In the second round of execution of forward FTPN, transition $t_{2}$ is first fired to simulate the malfunction of relay $R_{2}$ and the result is compared to backward FTPN. The marking of the backward sub-FTPN2 can be shown by vector $M=\left(\begin{array}{lllllllll}P_{9} & P_{10} & P_{11} & P_{12} & P_{13} & P_{14} & P_{15} & P_{16} & P_{25}\end{array}\right)$, the last place is the fault section estimation and designated by $F$ in place $P_{25}$. Therefore, with receiving information from $R_{2}$ and $\mathrm{CB}_{2}$, the

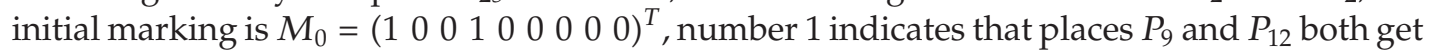
token and zero means otherwise. With this marking only transition $t_{11}$ is enabled and can fire. Transition $t_{12}$ is not enabled because the place with inhibitory arc connected to it is marked. Firing transition $t_{11}$ removes token from places $P_{9}$ and $P_{12}$ and deposites one token in the places $P_{11}$ and $P_{12}$. After firing this transition the new marking is $M_{1}=\left(\begin{array}{lllllll}0 & 0 & 1 & 1 & 0 & 0 & 0\end{array}\right)^{T}$. Having token in places $P_{11}$ and $P_{12}$ the transition $t_{14}$ is now enabled and can fire. Firing $t_{14}$

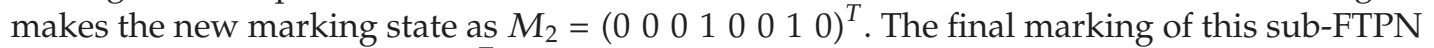

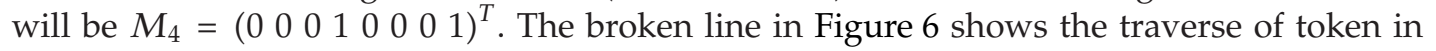
sub-FTPN2. Having delay time of $R_{2}$ and $\mathrm{CB}_{2}$, the initial fuzzy timestamps would be as in Figure 3.

With these fuzzy timestamps at the places $P_{9}$ and $P_{12}$, first fuzzy enabling time of transition $t_{11}$ should be found.

$e_{11}(\tau)=$ latest $\left\{\pi_{r}(\tau), \pi_{b}(\tau)\right\}=$ latest $\{(0.1,0.2,0.2,0.3),(0.2,0.3,0.3,0.4)\}=$ $(0.2,0.3,0.3,0.4)$. Then, fuzzy occurrence time of $t_{11}$ is computed (see $\left.(3.2)\right): o_{11}(\tau)=$ 


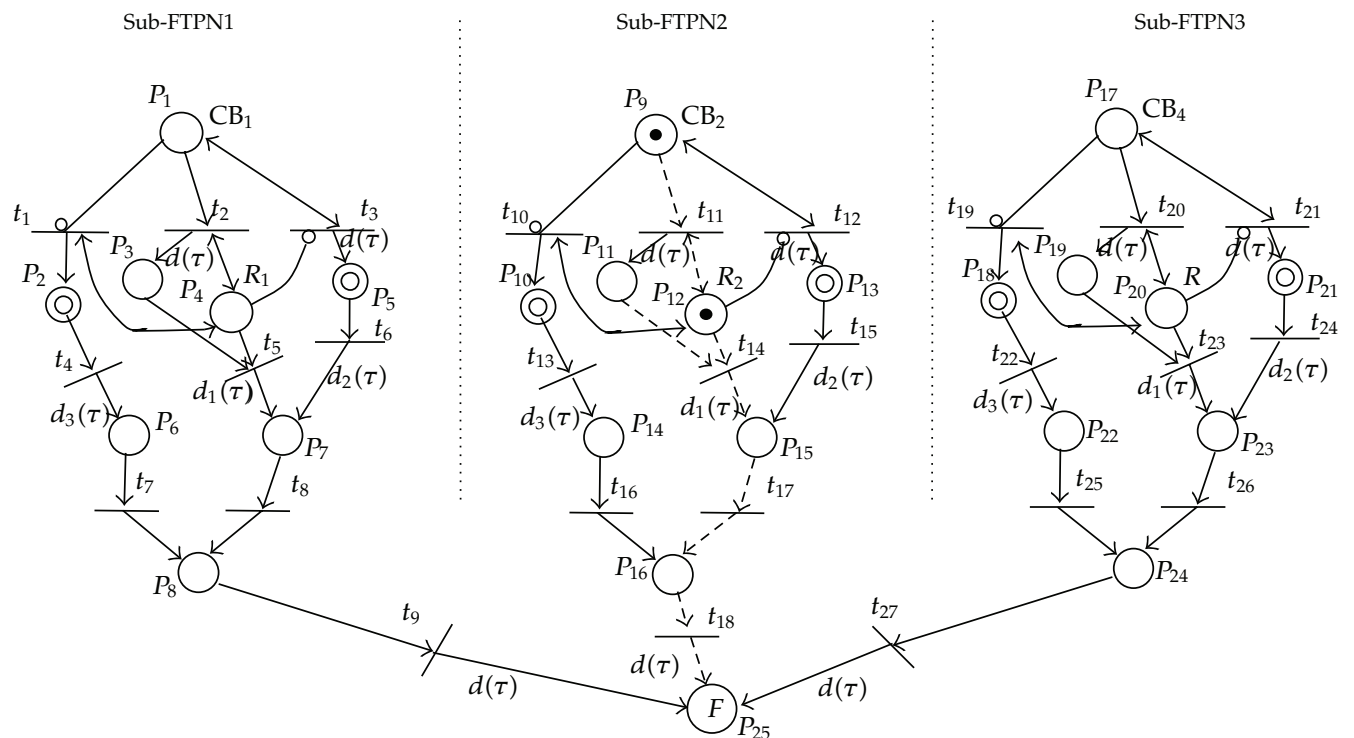

(a)

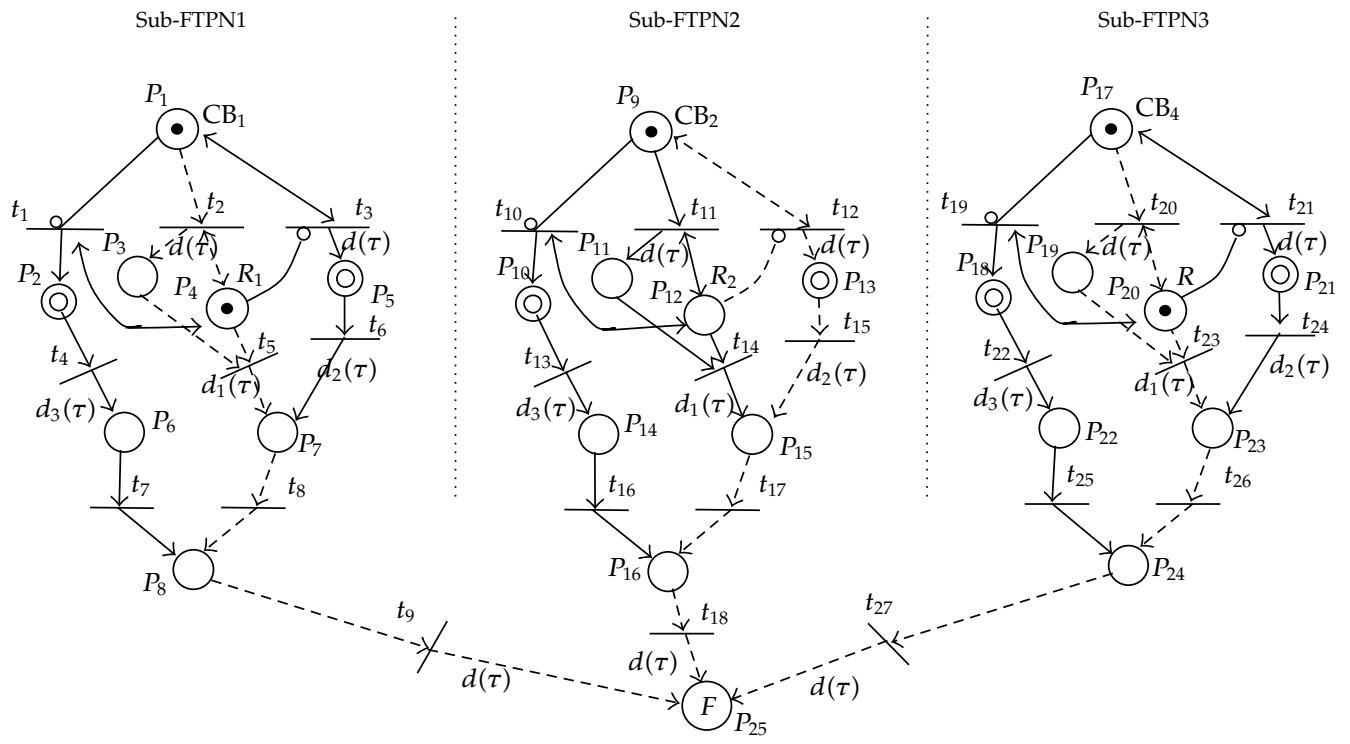

(b)

Figure 4: The backward FTPN model of Figure 1 for fault at point $F$ (shown by place $P_{25}$ ). $\mathrm{CB}_{2}$ and $R_{2}$ correspond to the main protection, and $\mathrm{CB}_{1}, R_{1}, \mathrm{CB}_{4}$, and $R_{4}$ correspond to the backup protection. (a) Information received from $R_{2}$ and $\mathrm{CB}_{2} . \sigma_{1}$ is the firing sequence of subFTPN2. $\sigma_{1}=M_{0}\left[t_{11}\right\rangle M_{1}\left[t_{14}\right\rangle M_{2}\left[t_{17}\right\rangle M_{3}\left[t_{18}\right\rangle M_{4}, M_{0}=\left(P_{9} P_{12}\right), M_{1}=\left(P_{11} P_{12}\right), M_{2}=$ $\left(P_{12} P_{15}\right), M_{3}=\left(P_{12} P_{16}\right), M_{4}=\left(P_{12} P_{25}\right)$. (b) Information received from $\mathrm{CB}_{2}, \mathrm{CB}_{1}, R_{1}, \mathrm{CB}_{4}$, and $R_{4} . \sigma_{1}, \sigma_{2}$, and $\sigma_{3}$ are firing sequence of sub-FTPN1, sub-FTPN2, and sub-FTPN3, respectively, $\sigma_{1}=M_{0}\left[t_{2}\right\rangle M_{1}\left[t_{5}\right\rangle M_{2}\left[t_{8}\right\rangle M_{3}\left[t_{9}\right\rangle M_{4}, \sigma_{2}=M_{5}\left[t_{12}\right\rangle M_{6}\left[t_{15}\right\rangle M_{7}\left[t_{17}\right\rangle M_{8}\left[t_{18}\right\rangle M_{9}, \sigma_{3}=$ $M_{10}\left[t_{20}\right\rangle M_{11}\left[t_{23}\right\rangle M_{12}\left[t_{26}\right\rangle M_{13}\left[t_{27}\right\rangle M_{14}, M_{0}=\left(P_{1} P_{4}\right), M_{1}=\left(P_{3} P_{4}\right), M_{2}=\left(P_{4} P_{7}\right), M_{3}=\left(P_{4} P_{8}\right)$, $M_{4}=\left(P_{4} P_{25}\right), M_{5}=\left(P_{9}\right), M_{6}=\left(P_{9} P_{13}\right), M_{7}=\left(P_{9} P_{15}\right), M_{8}=\left(P_{9} P_{16}\right), M_{9}=\left(P_{9} P_{25}\right), M_{10}=\left(P_{17} P_{20}\right)$, $M_{11}=\left(P_{19} P_{20}\right), M_{12}=\left(P_{20} P_{23}\right), M_{13}=\left(P_{20} P_{24}\right), M_{14}=\left(\begin{array}{ll}P_{20} P_{25}\end{array}\right)$. 


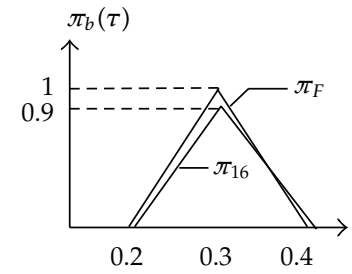

Figure 5: Comparison of two possibility distributions $\pi_{F}$ and $\pi_{16}$.

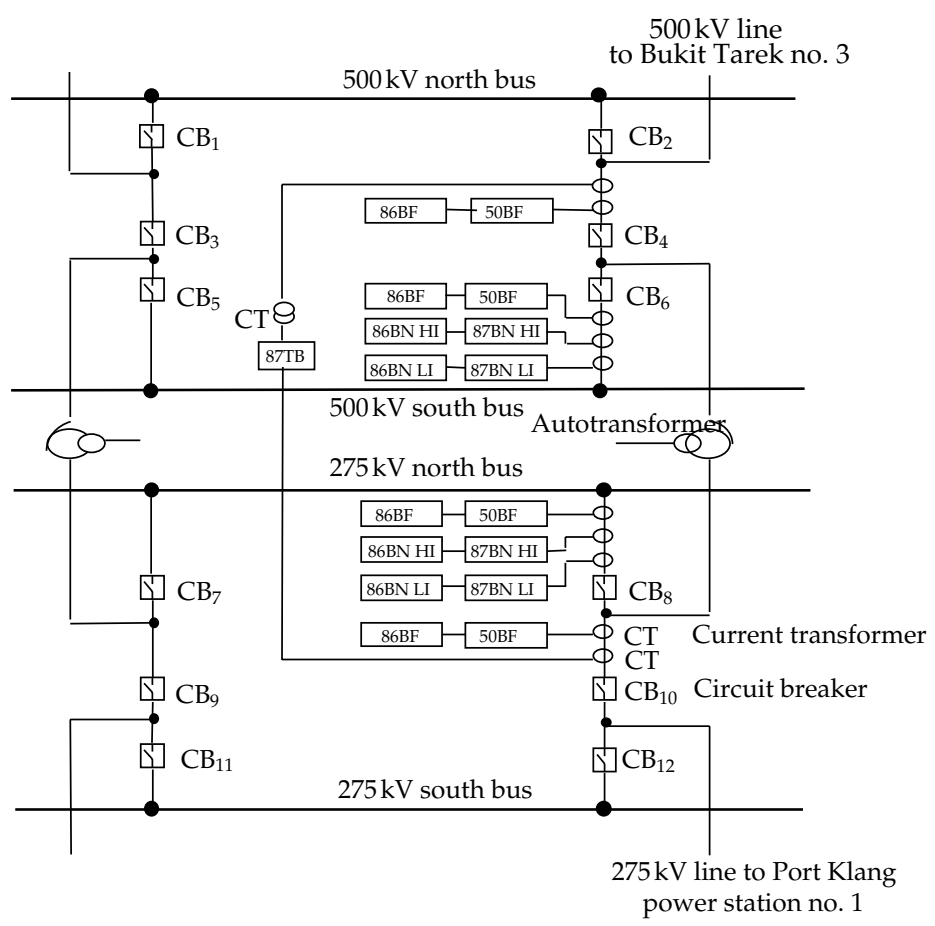

Figure 6: A simplified protection scheme of Kapar substation.

$\min \{(0.2,0.3,0.3,0.4)$, earliest $\{(0.2,0.3,0.3,0.4)\}\}=(0.2,0.3,0.3,0.4)$. Next fuzzy timestamp of place $P_{11}$ is calculated $(3.3)$ as $\pi_{11}(\tau)=o_{11}(\tau) \oplus d_{11}(\tau)=(0.2,0.3,0.3,0.4) \oplus$ $(0,0,0,0)=(0.2,0.3,0.3,0.4)$. Here it is assumed that the fuzzy delay time $d_{11}(\tau)$ is $(0,0,0,0)$.

This process is performed for firing sequence $\sigma_{1}$ until the final state of sub-FTPN2 (i.e., place $\left.P_{25}(F)\right)$. In this case, the fuzzy time function of place $F$ will be $\pi_{F}(\tau)=(0.2,0.3,0.3,0.4)$.

Having fuzzy timestamps of fault hypothesis in the backward FTPN, the fuzzy timestamps of final marking in the forward FTPN (see Figure 2) are to be computed. The following are assumed:

$\pi_{1}(\tau)=(0,0,0,0)$, means that token in place $P_{1}$ is immediately available. $\pi_{5}(\tau)=$ $(0.1,0.2,0.2,0.3)$ and $\pi_{9}(\tau)=\pi_{11}(\tau)=(0.3,0.4,0.4,0.5)$.

Having token in place $P_{2}$, the fuzzy enabling time of transition $t_{3}$ is

$$
e_{3}(\tau)=\operatorname{latest}\left\{\pi_{0}(\tau), \pi_{5}(\tau)\right\}=(0.1,0.2,0.2,0.3) .
$$


To compute fuzzy occurrence time of transition $t_{3}$, the earliest enabling time of $t_{2}$ and $t_{3}$ is found first as follows.

earliest $\left\{e_{3}(\tau) \oplus 0.9(0.01,0.01,0.03,0.03), e_{2}(\tau) \oplus 0.1(0.25,0.25,0.4,0.4)\right\}=$ earliest $\{0.1$, $0.2,0.2,0.3) \oplus 0.9(0.01,0.01,0.03,0.03),(0,0,0,0) \oplus 0.1(0.25,0.25,0.4,0.4)\}=$ $\max (0.9,0.1), \min (0.11,0.25), \min (0.21,0.25), \min (0.21,0.4), \min (0.31,0.4)\}=$ $0.9(0.11,0.21,0.21,0.31)$.

Therefore, the fuzzy occurrence of $t_{3}$ is computed as follows:

$o_{3}(\tau)=\min \left\{e_{3}(\tau) \oplus 0.9(0.01,0.01,0.03,0.03)\right.$, earliest $\left\{e_{3}(\tau) \oplus 0.9(0.01,0.01,0.03\right.$, $\left.0.03), e_{2}(\tau) \oplus 0.1(0.25,0.25,0.4,0.4\}\right\}=\min \{(0.1,0.2,0.2,0.3) \oplus 0.9(0.01,0.01,0.03$, $0.03)$, earliest $\left\{e_{3}(\tau) \oplus 0.9(0.01,0.01,0.03,0.03), e_{2}(\tau) \oplus 0.1(0.25,0.25,0.4,0.4\}\right\}=$ $\min \{0.9(0.11,0.21,0.21,0.31), 0.9(0.11,0.21,0.21,0.31)\}=0.9(0.11,0.21,0.21,0.31)$.

Now fuzzy timestamp of place $P_{3}$ is found as follows:

$\pi_{3}(\tau)=o_{3}(\tau) \oplus d_{3}(\tau)$, where $d_{3}(\tau)$ is fuzzy delay time from transition $t_{3}$ to place $P_{3}$. $\pi_{3}(\tau)=0.9(0.11,0.21,0.21,0.31) \oplus(0,0,0,0)=0.9(0.11,0.21,0.21,0.31)$. Then the fuzzy occurrence transition $t_{4}$ would be as follows:

$$
\begin{aligned}
o_{4}(\tau)=e_{4}(\tau) & =\pi_{3}(\tau)=0.9(0.11,0.21,0.21,0.31) \\
\pi_{16}(\tau)=o_{4}(\tau) \oplus d_{4}(\tau) & =0.9(0.11,0.21,0.21,0.31) \oplus(0.1,0.1,0.1,0.1) \\
= & 0.9(0.21,0.31,0,31,0,41) .
\end{aligned}
$$

\section{Comparison of Two Fuzzy Timestamps}

At this stage of diagnosis the comparison of two fuzzy timestamps $\pi_{F}$ and $\pi_{16}$ derived from forward and backward FTPN is to be evaluated. Refer to [13]. The possibility of $\pi_{F}=\pi_{16}$ may be found as follows (see Figure 5):

$$
\prod\left(\pi_{F}=\pi_{16}\right)=\min \left\{\prod\left(\pi_{F} \leq \pi_{16}\right) \prod\left(\pi_{16} \leq \pi_{F}\right)\right\}=\min (0.9,1)=0.9 .
$$

If the threshold value, $\lambda$, is assumed to be $\lambda=0.8$, therefore it is concluded that a fault has occurred at point $F$ and relay $R_{2}$ and breaker $\mathrm{CB}_{2}$ have operated correctly.

\section{Application}

Figure 6 depicts one of the existing Malaysian substations, the so-called Kapar substation. It consists of two breaker and half systems $(500 \mathrm{kV}$ and $275 \mathrm{kV})$, which are connected by autotransformer. Since the complete protection scheme of the substation is complex, only simplified protection version will be used for one of the buses, say $275 \mathrm{kV}$ north bus.

At the $275 \mathrm{kV}$ north bus—at the $\mathrm{CB}_{8}$ side — the following protective devices are used:

87BN HI: high impedance busbar relay (trips $86 \mathrm{~N} \mathrm{HI),}$

87BN LI: low impedance busbar relay (trips 86N LI),

50BF: breaker failure (trips $86 \mathrm{BF}$ ), 


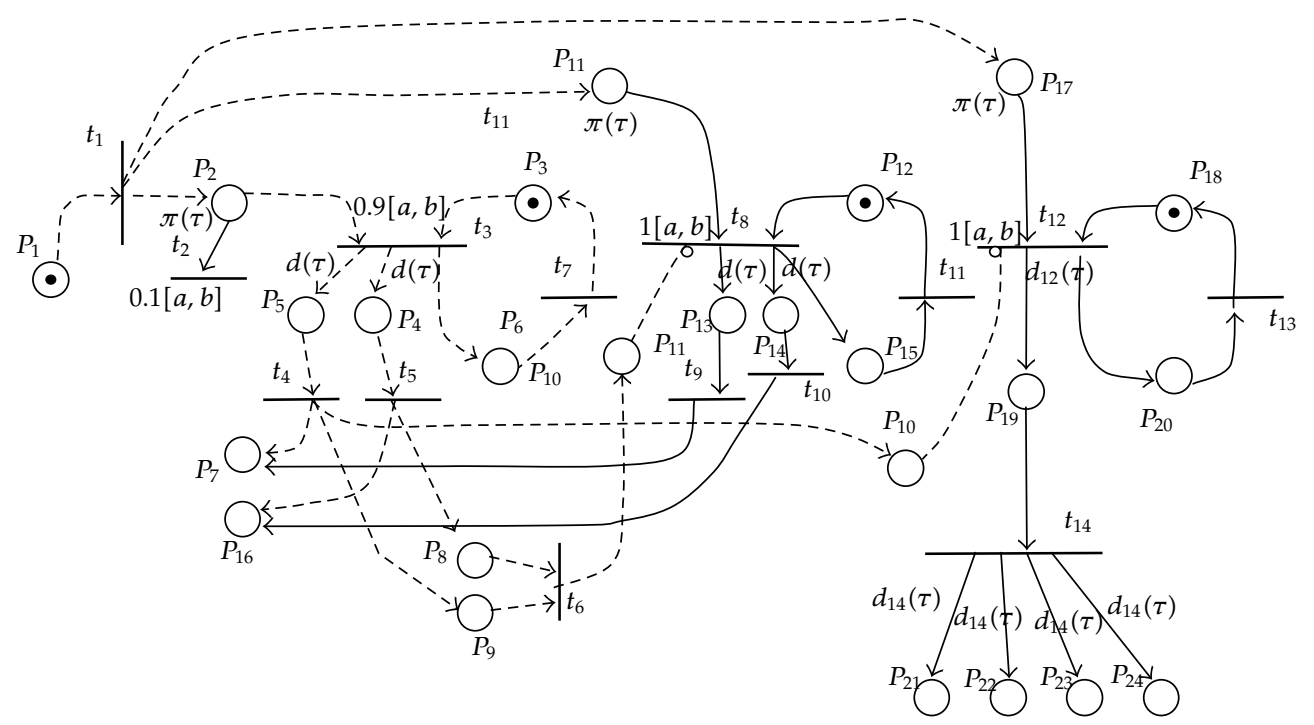

Figure 7: The forward FTPN model of protection scheme for fault at $275 \mathrm{kV}$ north bus bar of Kapar substation. The marking state of FTPN before occurrence of fault. The token in $P_{1}$ shows absence of fault, and firing transition $t_{1}$ indicates the occurrence of fault. Tokens in places $P_{3}, P_{12}$, and $P_{18}$ indicate the readiness of main, local backup, and breaker failure relays, respectively. The broken lines show the FTPN route in its firing sequences (in the case of correct operation of main relay and circuit break).

86BF: breaker failure lockout relay,

86BN HI: high impedance busbar lockout relay,

86BN LI: low impedance busbar lockout relay.

The same protection scheme is at the $500 \mathrm{kV}$ south bus. In addition, the autotransformer is protected by relay $87 \mathrm{~TB}$, which is a biased transformer differential relay.

Suppose a fault occurs at $275 \mathrm{kV}$ north bus of the Kapar substation. If the main relay (the busbar differential protection-87BN-HI) senses the fault and operates correctly, it then sends trip to $\mathrm{CB}_{8}$ and $\mathrm{CB}_{7}$ to isolate the busbar from fault. This scenario is modeled by the forward and backward FTPN and shown in Figures 7 and 8, respectively. If the main relay fails to operate, the local backup relay (87BN-LI) sends trip signal to the breakers $\mathrm{CB}_{8}$ and $\mathrm{CB}_{7}$. In the case of malfunction of circuit breaker $\mathrm{CB}_{8}$ the breaker failure relay will send trip signal to the circuit breakers $\mathrm{CB}_{7}, \mathrm{CB}_{4}, \mathrm{CB}_{6}$, and $\mathrm{CB}_{10}$. The broken lines in the forward FTPN models (Figure 7) show the route of FTPN in their firing sequences corresponding to their protection scheme.

In the backward FTPN models the token in place $F$ shows the estimated fault section, which in this case is $275 \mathrm{kV}$ busbar. This estimated fault section hypothesis in turn is compared with its relevant forward FTPN models. The procedure is similar as the one explained in Section 6.

\section{Conclusions}

A new model-based reasoning for power system fault diagnosis is proposed in this paper. It is based on fuzzy timing Petri net. It is believed that this proposed system could cover 


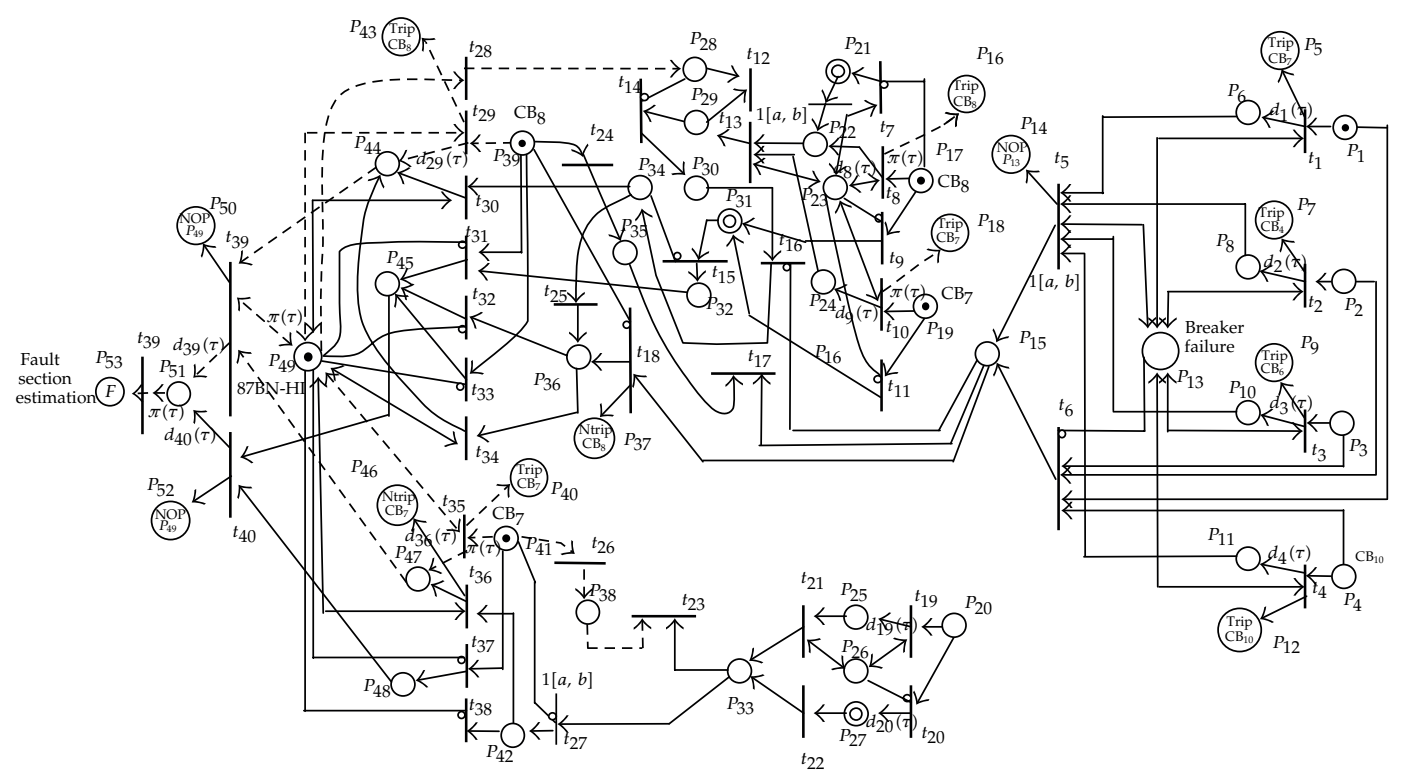

Figure 8: The backward FTPN model of Figure 6 for fault at $275 \mathrm{kV}$ north busbar of Kapar substation. The marking state of FTPN indicates the postfault condition. Places $P_{49}, P_{23}, P_{26}$, and $P_{13}$ correspond to main, local backup, remote backup, and breaker-failure relay, respectively. Token in place $P_{49}$ indicates that the main relay (87BN HI) has been operated and its signal has been received. Receiving signal from circuit breaker $\mathrm{CB}_{8}$ is shown by a token in places $P_{17}$ and $P_{39}$. Receiving signal from circuit breaker and $\mathrm{CB}_{7}$ is shown by a token in places $P_{1}, P_{19}$, and $P_{41}$. The broken lines are the routes of the FTPN in its firing sequences.In the case of tripping circuit breakers, places designated by trip $C_{X}$ get token, and in the case of nonoperation of any $\mathrm{CB}$, places the indicated by Ntrip would get token. OP $P_{49}$ means that the main relay has operated and NOP $P_{49}$ means otherwise. Transitions $t_{12}, t_{17}$, and $t_{23}$ are sink transitions and would fire if the main relay and breakers $\mathrm{CB}_{8}$ and $\mathrm{CB}_{7}$ have operated, respectively.

drawbacks of the previously reported model-based systems. This is because this proposed system gets the advantages of some powerful tools and concepts for designing real-time systems such as Petri net for modeling parallel processing and power of fuzzy set theory to handle uncertainty and imprecision. This system is able to handle parallel processing, therefore reducing the inference processing time. On the other hand, it uses the timestamps of circuit breakers (CB) and relays, which causes the fault hypotheses to be reduced accordingly. Moreover, the uncertain information from $\mathrm{CB} s$ and relays is handled by this system, therefore causing the diagnosis of the faulted section area to be more precise.

\section{Acknowlegment}

The first author would like to thank UTM for providing finacial support for this research.

\section{References}

[1] T. Minakawa, Y. Ichikawa, M. Kunugi, K. Shimada, N. Wada, and M. Utsunomiya, "Development and implementation of a power system fault diagnosis expert system," IEEE Transactions on Power Systems, vol. 10, no. 2, pp. 932-939, 1995. 
[2] S. Ebron, D. L. Lubkeman, and M. White, "Neural network approach to the detection of incipient faults on power distribution feeders," IEEE Transactions on Power Delivery, vol. 5, no. 2, pp. 905-914, 1990.

[3] Y. Sekine, Y. Akimoto, M. Kunugi, C. Fukui, and S. Fukui, "Fault diagnosis of power systems," Proceedings of the IEEE, vol. 80, no. 5, pp. 673-683, 1992.

[4] X. Wang and T. Dillon, "A second generation expert system for fault diagnosis," International Journal of Electrical Power and Energy Systems, vol. 14, no. 2-3, pp. 212-216, 1992.

[5] T. Murata, "Petri nets: properties, analysis and applications," Proceedings of the IEEE, vol. 77, no. 4, pp. 541-580, 1989.

[6] K. L. Lo, H. S. NG, and J. Trecat, "Power System fault diagnosis using Petri nets," IEE Proceedings Generation, Transmission $\mathcal{E}$ Distribution, vol. 144, no. 3, pp. 231-236, 1997.

[7] C. L. Yang, A. Yokoyama, and Y. Sekine, "Fault Section estimation of power system using Colored and timed Petri nets," in Proceedings of the Expert Systems Application to Power System, pp. 321-326, 1993.

[8] A. Hertz and P. Fauquembergue, "Fault diagnosis at substations based on sequential event recorders," Proceedings of the IEEE, vol. 80, no. 5, pp. 684-688, 1992.

[9] J. L. P, de Sa and J. P. S. Paiva, "Design and verification of concurrent switching sequences with Petri nets," IEEE Transactions on Power Delivery, vol. 5, no. 4, pp. 1766-1772, 1990.

[10] F. Wang and J. Tang, "Modeling of a transmission line protection relaying scheme using Petri nets," IEEE Transactions on Power Delivery, vol. 12, no. 3, pp. 1055-1060, 1997.

[11] S. M. Chen, J. S. Ke, and J. F. Chang, "Knowledge representation using fuzzy Petri nets," IEEE Transactions on Knowledge and Data Engineering, vol. 2, no. 3, pp. 311-319, 1990.

[12] Y. Zhou and T. Murata, "Pert net model with Fuzzy timing and Fuzzy-metric temporal logic," International Journal of Intelligent Systems, vol. 14, pp. 719-745, 1999.

[13] S. Ribarić, B. D. Bašić, and L. Maleš, "An approach to validation of fuzzy qualitative temporal relations," in Proceedings of the 24th International Conference on Information Technology Interfaces (ITI' 02), pp. 223-228, Cavtat, Croatia, 2002.

[14] A. A. M. Zin, A. M. Yousf, A. Tavakoli, and K. L. Lo, "Power System Fault diagnosis Using Artificial Intelligent," in Student Conference on Research and Development (SCOReD '01), Kuala Lumpur, Malaysia, February 2001.

[15] A. A. M. Zin, A. M. Yousf, A. Tavakoli, and K. L. Lo, "Power system fault diagnosis using integrated time petri net and expert system," in International Power Engineering Conference (IPEC '01), Singapore, May 2001.

[16] A. T. Ghainani, Intelligent system for power system fault diagnosis using Fuzzy-timing Petri net-based expert system [M.S. thesis], Department of Electrical Engineering, University Teknologi Malaysia, Malaysia, 2001. 


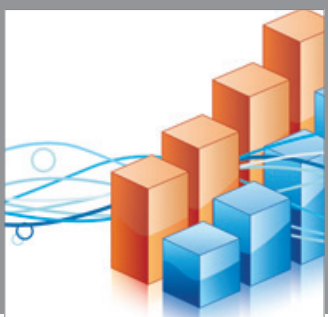

Advances in

Operations Research

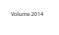

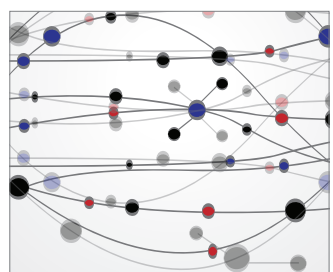

\section{The Scientific} World Journal
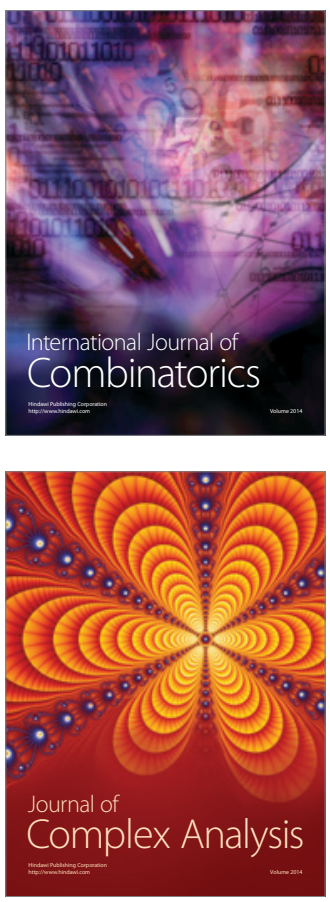

International Journal of

Mathematics and

Mathematical

Sciences
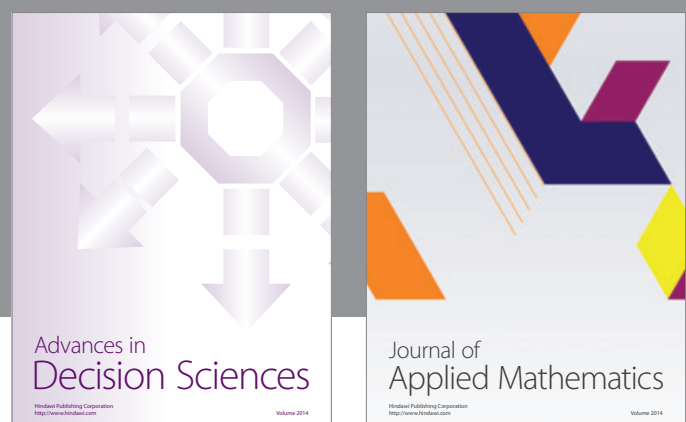

Journal of

Applied Mathematics
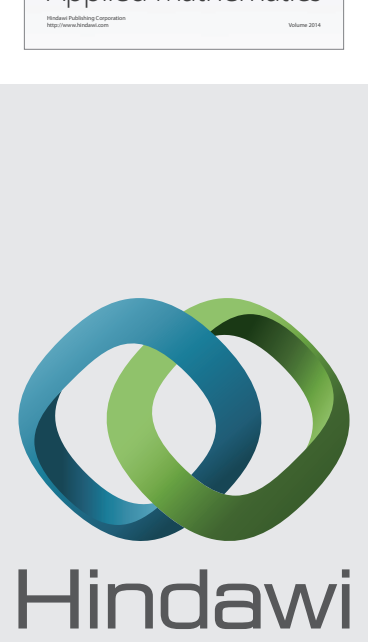

Submit your manuscripts at http://www.hindawi.com
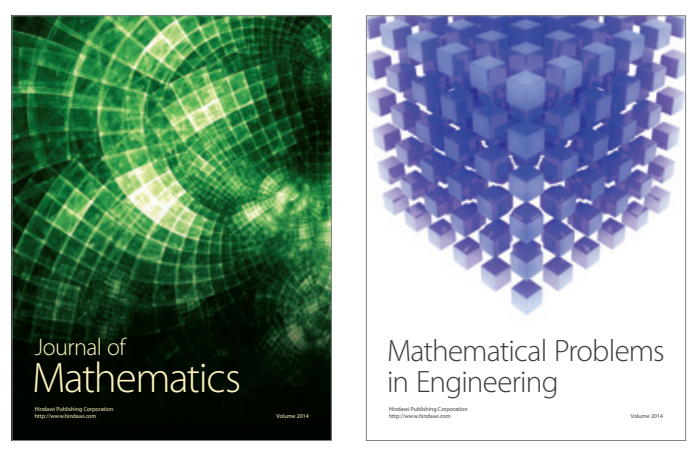

Mathematical Problems in Engineering
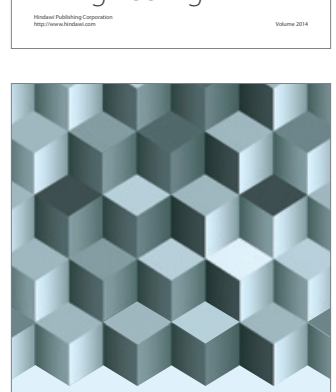

Journal of

Function Spaces
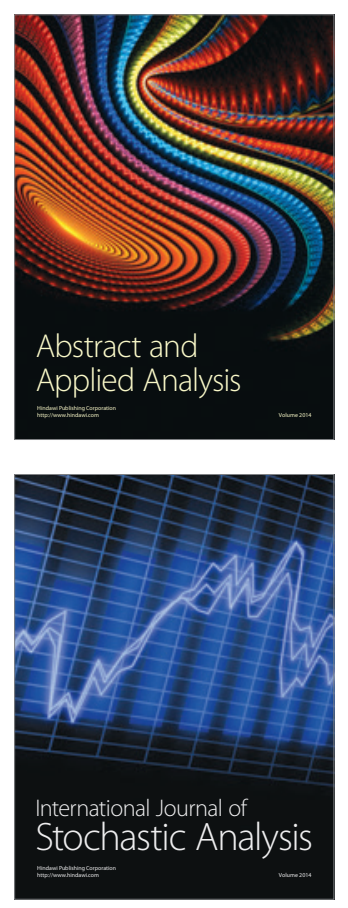

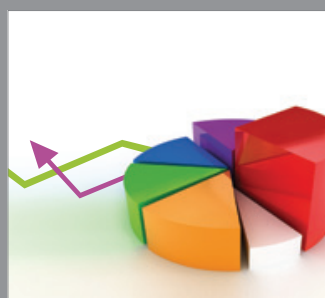

ournal of

Probability and Statistics

Promensencen
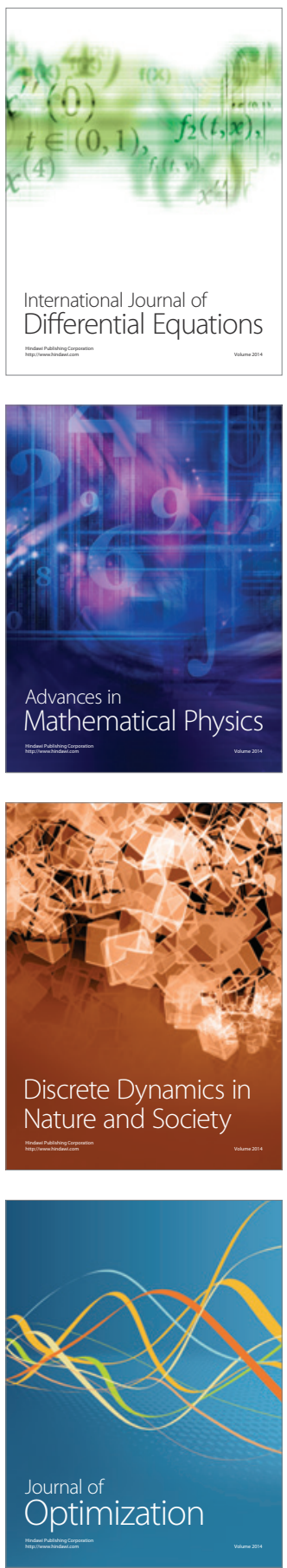